\title{
Amplitude growth of atmospheric gravity waves obtained from lidar and airglow image measurements.
}

Fábio Vargas (fabio@laser.inpe.br), INPE, Brazil

Gotao Yang, INPE, Brazil

Paulo Batista, INPE, Brazil

Delano Gobbi, INPE, Brazil

Copyright 2007, SBGf - Sociedade Brasileira de Geofísica

This paper was prepared for presentation at the $10^{\text {th }}$ International Congress of The Brazilian Geophysical Society held in Rio de Janeiro, Brazil, 19-22 November 2007.

Contents of this paper were reviewed by the Technical Committee of the $10^{\text {th }}$ International Congress of the Brazilian Geophysical Society and do not necessarily represent any position of the SBGf, its officers or members. Electronic reproduction, or storage of any part of this paper for commercial purposes without the written consent of the Brazilian Geophysical Society is prohibited.

\section{Abstract}

Amplitude growth rate of monochromatic gravity waves were estimated and compared in this study by using multiple instrument measurements carried out in Brazil. Wave dynamic parameters (vertical wavelength, horizontal wavelength, period, and wave amplitude) were obtained from sodium density profiles of lidar observations in São José dos Campos $\left(23^{\circ} \mathrm{S}, 46^{\circ} \mathrm{W}\right)$, while multiple airglow layer imaging provided brightness fluctuations amplitudes and parameters of waves over Cachoeira Paulista $\left(23^{\circ} \mathrm{S}, 4^{\circ} \mathrm{W}\right)$. Vertical scales of gravity waves in lidar measurements were found to range from 2 to $10 \mathrm{~km}$, and their growth rates are consistent with dissipative waves. These same characteristics are also observed in waves detected through airglow images, but their vertical scales are larger $\left(\lambda_{z}>15 \mathrm{~km}\right)$. Despite the fact that both instruments present complementary information, the general saturated/damped behavior is consistent with diffusive filtering processes imposing limits to the amplitude growth of the observed waves, i.e., the atmospheric diffusivity acts on the wave in order to reduce its amplitude.

\section{Introduction}

Gravity waves (GWs) play an important role in atmosphere dynamics due to their ability to transport momentum and energy from the lower to the upper atmosphere. Their influence on the mesospheric region $(80-100 \mathrm{~km})$ include heating through the turbulence generated by breaking waves, transport and mixing of constituents, reversal of the zonal mean jets and mean flow acceleration through momentum flux transference to the media, modifying the dynamical conditions at those altitudes [Fritts and Alexander, 2003; Fritts et al., 2006].

Freely propagating GWs (no dissipative waves) increase their amplitudes as $\sim \exp [\alpha z]$, where $\alpha=1 / 2 \mathrm{H}$ is the growth rate of freely propagating $\mathrm{GWs}, \mathrm{z}$ is the altitude and $\mathrm{H}$ is the atmospheric height scale. The wave amplitude increases in order to conserve kinetic energy in response to the atmospheric density decreasing with the altitude [Hines, 1960]. A typical value of $\mathrm{H}$ is $\sim 6 \mathrm{~km}$, and a wave generated at an altitude of $10 \mathrm{~km}$ is expected to present an amplitude $\sim 349$ times larger in mesosphere region $(\sim 90 \mathrm{~km})$ than that measured at lower altitudes.

Frequently, instability processes (i.e. convective and/or dynamical) or diffusion (atmospheric viscosity) impose limits to the amplitude of GWs, and departures from the factor $\alpha$ can be observed, indicating that the wave is being dissipated. Swenson et at. [2003] have investigated high frequency GWs ( $<1$ hour) disturbing the mesopause temperature by using wind/temperature lidar measurements. They have shown that GWs are basically saturated to over damped bellow $100 \mathrm{~km}$ of altitude, while unsaturated to freely propagating above this level. Also, Taori et al. [2007] have shown that small period waves $(<12 \mathrm{~h})$ observed in rotational temperature of $\mathrm{OH}(6-2)$ and $\mathrm{O}_{2}(0-1)$ emissions tend to be strongly dissipated throughout the year. GW characterization has been carried out using simultaneous measurements of the airglow intensity and temperatures by Reisin and Scheer [1996], where simultaneous measurements of the $\mathrm{OH}$ and $\mathrm{O}_{2}$ emission layers were used to infer the wave growth and dissipation. It was reported a high degree of variability in the wave amplitude growth within the short altitude range of $7 \mathrm{~km}$ (i.e. the spatial separation between $\mathrm{OH}$ and $\mathrm{O}_{2}$ layer centroids).

In this paper, we use two different instruments ( $\mathrm{Na}$ lidar and imager) for estimating wave amplitudes and the growth rates of GWs modulating the atmospheric fields at different altitudes in the mesopause region. These instruments sample different ranges of the GW spectra, providing complementary information about GW modes present in $\mathrm{Na}$ density and airglow intensity observations. The obtained results also give insights about the limiting processes taking place in response to wave amplitude increasing in the atmosphere.

\section{Method}

GW intrinsic parameters, amplitudes and growth rates were obtained from lidar and imager data in this study. Once both instruments provide wave amplitudes at different altitudes, growth rates may be estimated by $\beta=\ln \left[A_{2} / A_{1}\right] / \Delta z$, where $A_{2}$ and $A_{1}$ are the wave amplitudes at the altitude levels 2 and 1 , respectively, and $\Delta z$ is the distance between these levels. Here we refer $\beta$ as the growth rates of monochromatic waves generically, in order to distinguish from $\alpha$, the growth rate of freely propagating waves (no dissipative waves).

A lidar located in São José dos Campos $\left(23^{\circ} \mathrm{S}, 46^{\circ} \mathrm{W}\right)$ provided sodium vertical profiles, from where 45 monochromatic GWs were observed from 1994 to 2004. $\mathrm{Na}$ lidar measurements have been described by Simonich et al. [1979] and Clemesha et al. [1992], while the GW 
parameter estimation from lidar measurements have been carried out by Yang et al. [2007].

Fig. 1 gives an idea of how the monochromatic waves are identified from sodium lidar data. Fig. 1(a) shows a temporal series of vertical sodium profiles from 75-110 $\mathrm{km}$, with temporal (spatial) resolution of $3 \mathrm{~min}(250 \mathrm{~m})$. The sodium density profiles are first spatially and temporally low-pass filtered with a cutoff of about $1.5 \mathrm{~km}$ and 20 minutes, respectively. Coherent downward phase progression can be seen in the Fig. 1. Fig. 1(b) shows a single [Na] profile superposed to an unperturbed profile. The relative wave amplitude perturbing the $\mathrm{Na}$ sodium layer is given in Fig. 1(c), showing the wave is decreasing amplitude as it propagates upward. It was found in this case a wave presenting $\lambda_{z}=4.6 \mathrm{~km}$, amplitude of $2.46 \%$ (at $90 \mathrm{~km}$ ) and the inverse of the growth rate $1 / \beta=-24 \mathrm{~km}$. Wave periods, horizontal wavelengths and phase velocities can be also estimated by using the method described by Yang et al. [2007].

A multicolor imager operating at Cachoeira Paulista $\left(23^{\circ} \mathrm{S}, 45^{\circ} \mathrm{W}\right)$ provided images of the mesospheric airglow layers during 1999, 2000, 2004 and 2005. Images of $\mathrm{OH}$, $\mathrm{O}_{2}$ and $\mathrm{O}\left({ }^{1} \mathrm{~S}\right)$ emissions were analyzed and $\mathrm{GWs}$ which were present in two or three layers simultaneously were submitted to spectral processing in order to deduce wave amplitudes, growth rates and horizontal parameters (horizontal wavelength, phase differences, propagation direction, phase speed, period, etc). $52 \mathrm{GW}$ wave events were identified during the period of observations.

Fig. 2 illustrates how wave parameters and amplitudes are obtained from airglow images. There are three images recorded simultaneously of the $\mathrm{OH}, \mathrm{O}_{2}$ and $\mathrm{O}\left({ }^{1} \mathrm{~S}\right)$ emissions in the panel (a). Once the same GW is perturbing all three layers, straight lines are drawn perpendicularly to the wave fronts in each image and pixels along these lines are extracted and plotted [Fig. 2 (b)]. The spatial series are then subjected to spectral analysis [Fig. 2(c)] and horizontal wavelength and amplitudes are calculated. Small phase deviations observed for different emissions are caused by slight different acquisition times of each image.

The location of a spectral peak in the amplitude periodogram gives the horizontal wavelength the disturbing $\mathrm{GW}$, while integrating bellow the peak gives the relative wave amplitude in each layer. Because the vertical distance among the airglow layers are well known, growth rates are straightforward obtained. Spectral analysis of the spatial series extracted from Fig. 2(a) reveals a wave with horizontal wavelength of $\sim 40$ $\mathrm{km}$, period of $\sim 30$ minutes, propagation direction of $160^{\circ}$, observed phase speed of $\sim 20 \mathrm{~m} / \mathrm{s}$, and amplitude of $15 \%$, $7 \%$ and $5 \%$ in $\mathrm{OH}, \mathrm{O}_{2}$ and $\mathrm{O}\left({ }^{1} \mathrm{~S}\right)$ layers, respectively, indicating a dissipative wave.

\section{Results and Discussion}

It was possible to identify $45 \mathrm{GW}$ events from analysis of ten years of sodium density profiles recorded by lidar, while $52 \mathrm{GW}$ events were obtained from analysis of 4 years of airglow images. Essentially, these two instruments sampled distinct ranges of the GW spectra. Vertical scales accessed from lidar measurements are limited by the sodium layer thickness $(\sim 15 \mathrm{~km})$, and the shortest vertical wavelength is basically limited by the signal shot noise [Gardner and Voelz, 1987]. For this reason, waves identified in lidar data presented vertical wavelengths $\left(\lambda_{z}\right)$ ranging from $2.38 \mathrm{~km}$ to $9.34 \mathrm{~km}$, with most of these waves $(\sim 40 \%)$ ranging from 3 to $4 \mathrm{~km}$. Observed wave periods ( $\mathrm{T}$ ) ranged from $63 \mathrm{~min}$ to $\sim 20$ hours, with maxima occurrence $(66 \%)$ in the $100-300$ min bin. GWs in lidar measurements presented long horizontal scales $\left(31.6<\lambda_{x}<1887 \mathrm{~km}\right)$, but with a tendency of dominance of waves presenting $\lambda_{x}<200 \mathrm{~km}$. Wave amplitudes ranged from $0.77 \%$ to $8.4 \%$ of the ambient density, with an average value of $2.7 \%$.

The wave vertical wavelengths accessed by imager measurements are larger than the layer thickness (8-10 $\mathrm{km})$. Because the observed airglow intensity is given by the vertical integration of the volume emission rate of the emission, short vertical scale waves $(<15 \mathrm{~km})$ suffer interference inside the layer, and the wave intensity perturbation is strongly attenuated for ground based observations. Imagers are able to observe short period waves ( $\mathrm{T}<1$ hour) and fast phase velocities $(>40 \mathrm{~m} / \mathrm{s})$. The horizontal wavelength $\left(\lambda_{x}\right)$ accessed by this instrument are upper limited by the field of view (FOV) and lower limited by the spatial resolution of pixels $(\delta \mathrm{s} \sim 1 \mathrm{~km} /$ pixel in this study). Spectral analysis of the events observed in this study showed $\lambda_{x}$ ranging from $\sim 14$ to $\sim 78 \mathrm{~km}$. The analysis of spatial series revealed wave amplitude in intensity ranging from $0.6 \%-15 \%$ for the $\mathrm{OH}, 0.5 \%-8.5 \%$ for $\mathrm{O}_{2}$, and $0.5 \%-8.5 \%$ for $\mathrm{O}\left({ }^{1} \mathrm{~S}\right)$ emissions, respectively.

Fig. 3 shows histograms of the amplitude growth rate $(\beta)$ for waves observed in both imager and lidar data. Positive values of $\beta$ (under saturated region) indicate amplification, while negative values (over damped region) indicate decreasing wave amplitudes. Values of $\beta$ close to zero indicate that the amplitude does not change much as the wave propagates upward. Also, it is considered here that waves presenting $\beta>\sim 7$ are freely propagating waves, i. e, their amplitude increases as $\sim \exp [\alpha z]$.

Growth rates obtained from lidar measurements present $48.9 \%$ of negative values and $51.1 \%$ of positive values, showing a symmetric $\beta$ distribution. It is observed a maxima occurrence of waves in the range of $0<\beta<2$ (under saturated region) that represents $24 \%$ of the wave events observed in $\mathrm{Na}$ lidar profiles. Those waves presented amplification, but not as fast as $\sim \exp [\alpha z]$. It is also observed that $\sim 35.6 \%$ of waves in lidar profiles are close of being saturated $(\beta \sim 0)$.

For imager data, $\beta$ shows $61.5 \%$ of negative and $38.5 \%$ of positive values, indicating larger amount of over damped events. About $51.6 \%$ of imager waves show strong attenuation $(\beta<-6)$, while only $\sim 9 \%$ of the waves observed in lidar dataset have similar growth rates. Also, $\sim 15.4 \%$ of the waves are close to the saturation limit $(\beta \sim 0)$, in contrast with lidar-viewed GWs. The maxima occurrence of imager-viewed GWs $(15.4 \%)$ is located in the interval of $-10<\beta<-8$ (over damped region).

While freely propagating waves $(\beta>7)$ correspond to $8.9 \%$ and $11.5 \%$ of the events observed in lidar and imager, respectively, about $90 \%$ of waves observed in both instruments show dissipative behavior (departures from 
the growth rate $\alpha$ ). The wave energy transferred to the media due to dissipative wave processes may cause several effects in the atmosphere, as mean flow acceleration and local heating. In general hydrodynamic instabilities and diffusion processes are responsible to limit the wave amplitude.

The linear saturation theory (LST) predicts that the wave amplitude will reach the saturation limit when the horizontal perturbation velocity ( $u$ ') equals the intrinsic horizontal phase velocity of the wave $\left(c_{i}\right)$. The amplitude is then limited by convective or shear instabilities [Dewan and Good, 1986]. On the other hand, the diffusive filtering theory (DFT) states that waves will be severely damped by diffusion when the effective vertical diffusion velocity $(\mathrm{mD})$ of the particles experiencing the wave motion exceeds the vertical phase velocity of the wave $\left(\omega \mathrm{m}^{-1}\right)$, where $D, m$ and $\omega$ are the total effective atmospheric diffusivity, the vertical wavenumber and the wave frequency, respectively [Gardner, 1994]. In this meaning, waves presenting $\omega>m^{2} D$ propagate without attenuation, while waves $\omega<m^{2} D$ are removed from the spectra by diffusion. Analysis performed by Yang et al. [2007] suggests that GWs observed in lidar measurements are in accordance with DFT, while rules out the predictions of LST. However, some observed wave events in the lidar dataset presented peculiar behavior, suggesting that processes other than diffusivity have to be considered in order to explain the observed wave amplitude characteristics and growth rates.

\section{Conclusions}

Atmospheric gravity waves observed in lidar and imager measurements were analyzed in this study. An amount of 45 monochromatic waves were identified in lidar data, while 52 waves were obtained in images of mesospheric airglow layers, respectively. The results showed that while each instrument samples a distinct region of the GW spectra, about $90 \%$ of the events (in both datasets) were associated with dissipative waves. Growth rate distributions are distinct for waves observed in different datasets. The maxima occurrence $(24 \%)$ of lidar-viewed waves is located in the under saturated region $(0<\beta<2)$, while the maxima occurrence of waves observed in the imager dataset $(15.4 \%)$ is located between $-10<\beta<-8$. Also, $51.6 \%$ of imager-viewed waves were find in the strong dissipation region $(\beta<-6)$ against only $\sim 9 \%$ of these type of waves in the lidar dataset. GWs observed in lidar density profiles support the diffusive filtering theory, which states the dissipation of wave energy is mainly due to diffusivity processes acting on the wave amplitude.

\section{References}

Clemesha B. R., D. M. Simonich, and P. P. Batista (1992), A long-time trend in the height of the atmospheric sodium layer: possible evidence for global change. Geophys. Res. Lett., 19, 457-460.

Dewan, E. M., and R. E. Good (1986), Saturation and the "universal" spectrum for vertical profiles of horizontal scalar winds in the atmosphere. J. Geophys. Res., 91, 2742-2748.

Fritts, D. C., and M. J. Alexander (2003) Gravity wave dynamics and effects in the middle atmosphere, Rev. Geophys., 41(1), 1003, doi:10.1029/2001RG000106.

Fritts, D. C., S. L. Vadas, K. Wan and J. A. Werne (2006) Mean and variable forcing of the middle atmosphere by gravity waves.J. Atmos. Sol.-Terr. Phys. 68(3-5): 247265.

[5] Gardner, C. S. (1994), Diffusive filtering theory of gravity wave spectra in the atmosphere, J. Geophys. Res., 99, 20,601-20,622.

Gardner, C. S., and D. G. Voelz (1987) Lidar studies of the nighttime sodium layer over Urbana, Illinois, 2. Gravity waves, J. Geophys. Res., 92, 4673-4693.

Hines, C. O. (1960). Internal atmospheric gravity waves at ionospheric heights. Can. J. Phys. 38(11): 1441-1481.

Reisin, E. R., and J. Scheer, (1996) Characteristics of atmospheric waves in the tidal period range derived from zenith observations of $\mathrm{O} 2$ (0-1) Atmospheric and $\mathrm{OH}(6-2)$ airglow at lower midlatitudes, J. Geophys. Res., 101(D16), 21223-21232, doi:10.1029/96JD01723.

Simonich, D. M., B. R. Clemesha, and V. W. J. H. Kirchhoff (1979), The mesospheric sodium layer at $23^{\circ} \mathrm{S}$ : nocturnal and seasonal variations, J. Geophys. Res., 84, $1543-1550$

Swenson, G. R., A. Z. Liu, F. Li and J. Tang (2003) High frequency atmospheric gravity wave damping in the mesosphere, Adv. Space Res., 32(5), 785-793.

Taori, A. ; A. Guharay, and M. J. Taylor (2007) On the use of simultaneous measurements of $\mathrm{OH}$ and $\mathrm{O} 2$ emissions to investigate wave growth and dissipation, Ann. Geophys., 25, 639-643,

Yang, G., B. Clemesha, P. Batista, and D. Simonich (2007) Lidar Study of the Characteristics of Gravity Waves in the Mesopause Region at a Southern LowLatitude Location, J. Atmos. Sol-Terr. Phys., manuscript. 

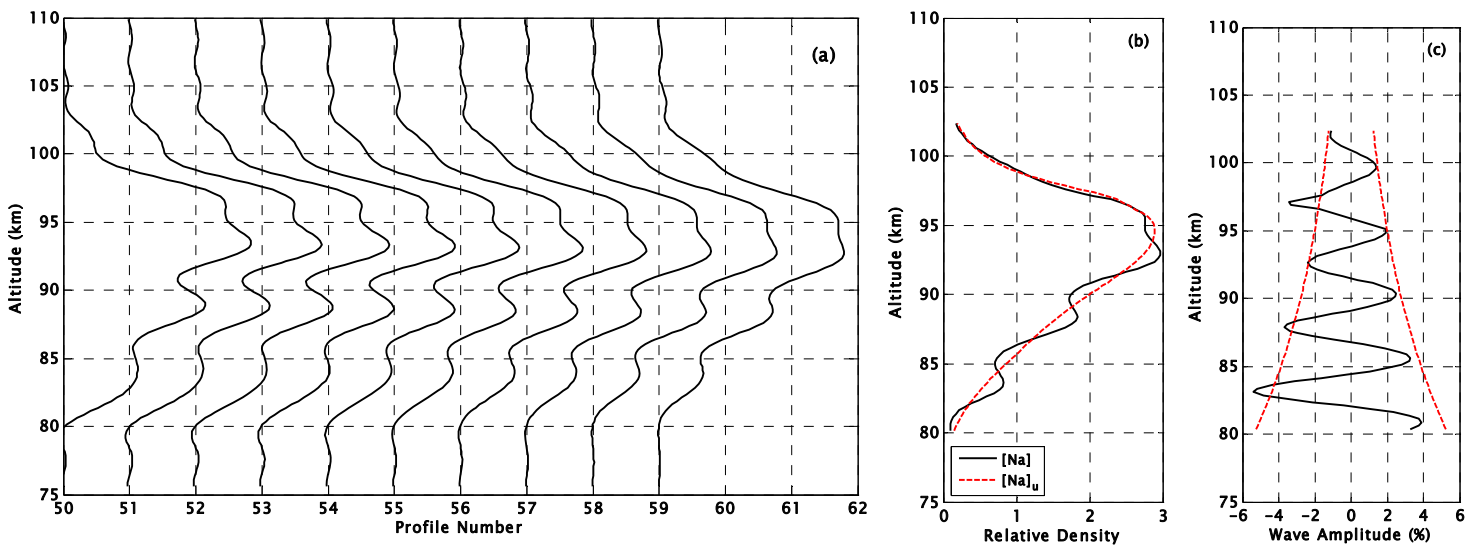

Figure 1 - (a) Observed sequence of sodium density profiles taken in May 30, 1996. (b) A single [Na] profile superposed by an assumed background profile. (c) Extracted wave amplitude from (b). It is remarkable the wave amplitude decreasing in altitude (i.e. $\beta<0)$.
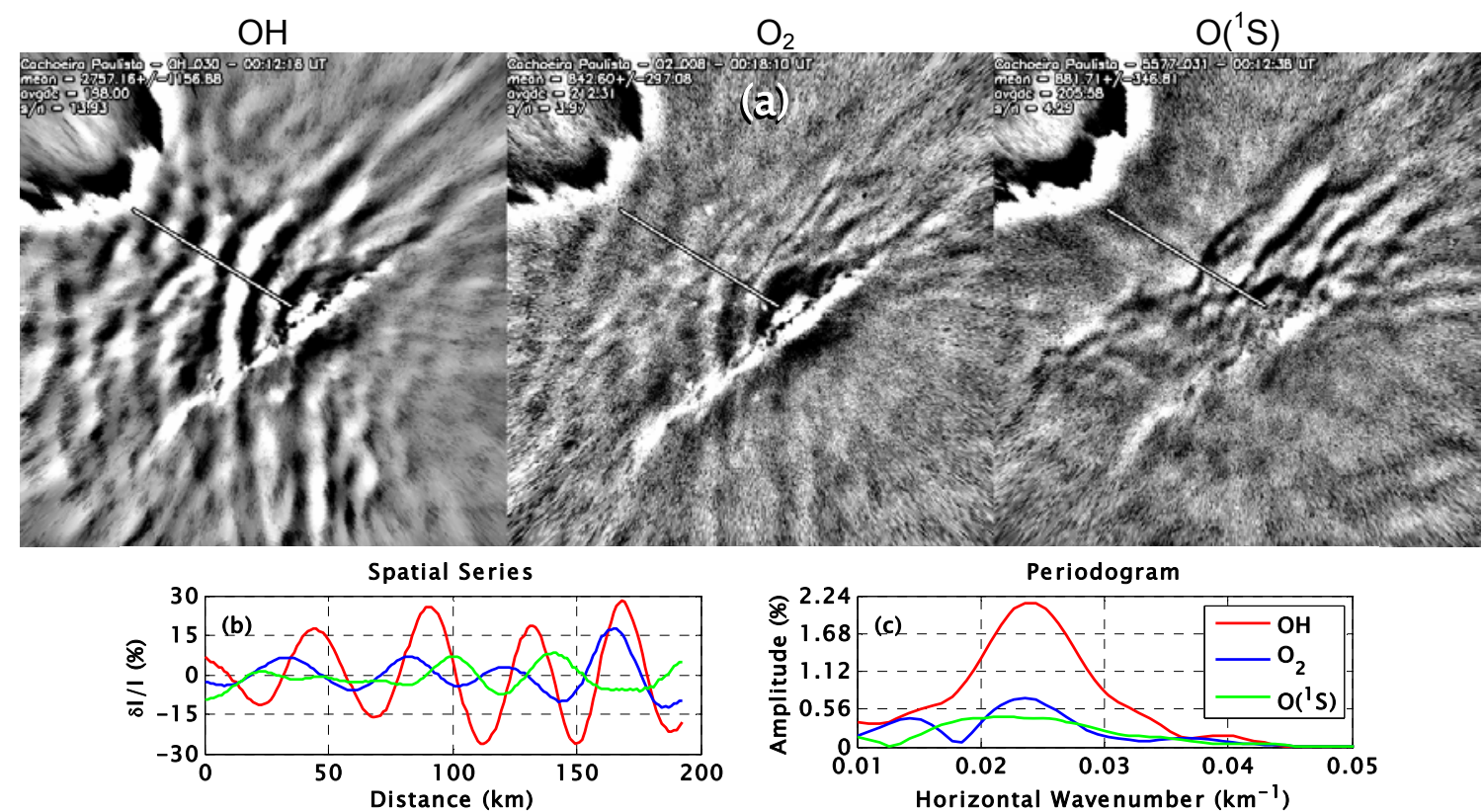

Figure $2-(\mathrm{a}) \mathrm{OH}, \mathrm{O}_{2}$ and $\mathrm{O}\left({ }^{1} \mathrm{~S}\right)$ images taken simultaneously in June 30, 2000, in Cachoeira Paulista, where a gravity wave is disturbing the emitted intensity of the layers. (b) Spatial series taken from each image when a line is traced perpendicularly to the front waves. (c) Spectral analysis of the spatial series giving the wave amplitude in each layer.

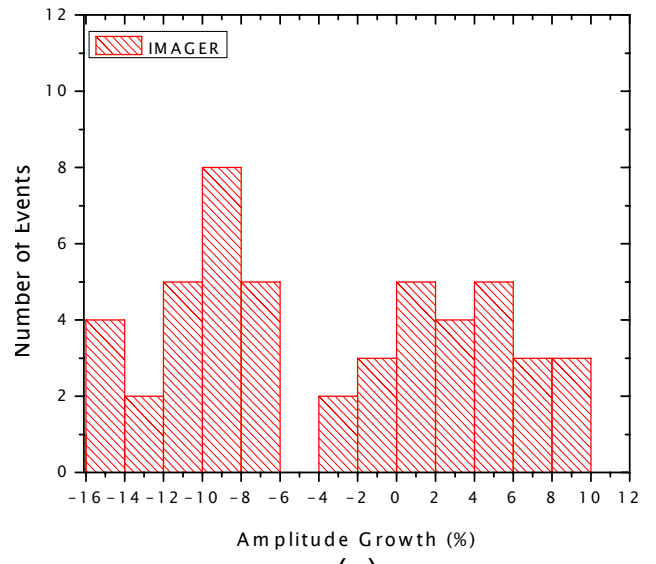

(a)

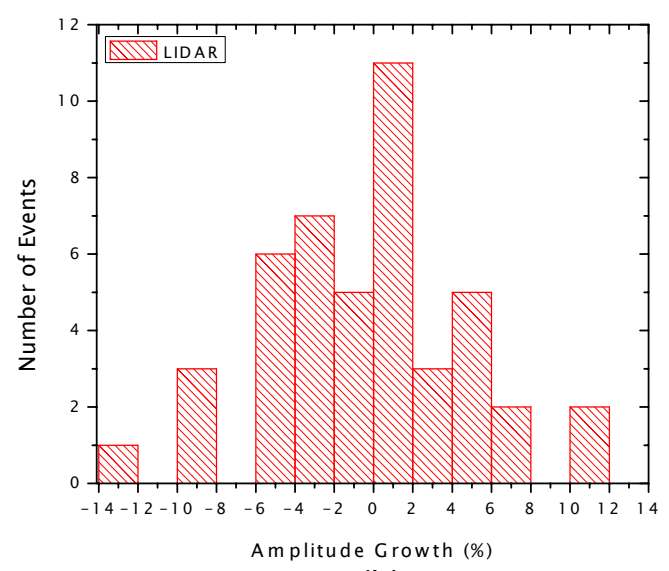

(b)

Figure 3 - Amplitude growth estimation of waves observed in (a) imager and (b) lidar data. Positive values of the growth rate indicate increasing amplitudes, while negative values indicate amplitude attenuation as the wave propagates upward. 